\title{
Water Disinfection By-products and the Risk of Congenital Anomalies in Kaunas
}

\author{
Violeta Kapustinskienė, Regina Gražulevičienė \\ Vytautas Magnus University, Lithuania
}

cross ${ }^{\text {ref }}$ http://dx.doi.org/10.5755/j01.erem.61.3.1147

(received in February, 2012, accepted in September, 2012)

\begin{abstract}
There is growing evidence from epidemiological studies that maternal exposure to increased drinking water chlorination with by-products - trihalometane (THM) may be associated with congenital anomalies. The aim of this study was to evaluate the impact of maternal exposure during pregnancy to individual THM internal dose effect on the risk of congenital anomalies.

We conducted a population-based prospective study of 3074 Kaunas residents births in 2007-2009, using THM exposure estimated as internal dose. We used multivariate logistic regression analysis and compared the risk of any congenital anomalies in four exposure categories of THM internal dose quartiles. In addition, we conducted a linear regression models analysis with adjustment for maternal physiology, lifestyle and sociodemographic characteristics.

Effect estimates based on total THMs and chloroform quartiles tended to increase congenital anomalies risk, but there was no statistically significant effect. However, we found dose-response relationships for the bromodichloromethane (BDCM) internal dose and risk for any congenital anomalies. The adjusted odds ratio for fourth quartile vs. first quartile was $1.5,95 \%$ CI $1.00-2.55$. The OR per every $0.01 \mu \mathrm{g} / \mathrm{d}$ increase in BDCM internal dose was $1.03,95 \%$ CI 1.00-1.07. Effect estimates associated with dibromochloromethane (DBCM) exposure levels also revealed statistically significant association with the congenital anomalies risk (OR 1.23, 95\% CI 1.02-1.48).

The present study suggests that prenatal exposure to total THM has tendency to increase the risk of any congenital anomalies, while exposure to BDCM and DBCM statistically represents a significant increase in the risk of any congenital anomaly.

Keywords: drinking water by-products, THM, internal dose, congenital anomalies.
\end{abstract}

\section{Introduction}

Water chlorination is a widely used and efficient method to reduce the occurrence of water-borne infectious diseases, and has been one of the most successful public health measures introduced in the 20th century. Disinfection by-products trihalomethanes (THMs) are a major group of water contaminants, and their role in causing adverse birth outcomes has been subject to extensive epidemiologic and toxicologic research and review (Bove et al. 2002; Butterworth 2005; Goebell et al. 2004; Nieuwenhuijsen et al. 2009; Tardiff et al. 2006;). Generally, the THMs, including chloroform, bromodicholormethane, dibromochlormethane, and bromoform are the most prevalent in chlorinated surface water (Nieuwenhuijsen et. al. 2000). A metaanalysis of the epidemiological studies has indicated that exposure to THMs may increase the risk of birth defects in general, especially neural tube and urinary tract defects (Hwang and Jaakkola 2003). A Swedish study provided some evidence of an elevated risk of cardiac defects (Cedergren et al. 2002), whereas Californian case-control study provided inconsistent results (Shaw et al. 2003). In a Norwegian nationwide cross-sectional study, the risk of specific birth defects was related to exposure to THMs (Hwang et al. 2002). A recent study in England and Wales reported that the risk of ventricular septal defects was associated with exposure to THMs while total brominated THM exposure in the first trimester of pregnancy did not trigger significant excess risks of congenital anomalies in the high-exposure categories. (Nieuwenhuijsen et al. 2008). 
A major challenge of these studies was the imprecision of exposure assessment from using aggregate municipal drinking water measures for classifying THM exposures. Most of the previous research has focused on exposure to concentrations of total THMs. As the researchers had no information on individual patterns of water consumption, showering, or bathing, the assigned category of THM exposure might not accurately reflect the actual THM uptake. Moreover, the epidemiological studies differed in the control of maternal characteristics that could also be associated with adverse pregnancy outcomes. Such differences present difficulties in making comparisons between research and generalised results.

Seeking to improve the estimation of THM exposure at the personal level, we conducted a prospective Kaunas pregnant women cohort study, which incorporated different routes of THMs uptake. This is the first epidemiological study that evaluates individual internal dose impact of THM constituents on congenital anomalies. Previously, we reported dose-response relationships for entire pregnancy and trimester-specific gestational THMs and chloroform internal dose for low birth weight and reduction in birth weight (Grazuleviciene et al. 2011).

In that case-control study we used individual maternal data from the prospective pregnant women cohort study to evaluate individual THM uptake during pregnancy and to assess the effect of internal dose of total THMs and individual THM on the risk of any congenital anomalies, adjusting for many important risk factors for congenital anomalies. Individual exposure to THM was estimated as the total internal dose based on the monitoring of drinking water THM levels and detailed water use behaviours.

\section{Materials and methods}

\subsection{Participant characteristics}

The epidemiological study in Kaunas city, Lithuania (KANC study) enrolled 4,161 pregnant women at the third month of pregnancy (2007-2009), residents of the municipality of Kaunas. The study ethics complied with the Declaration of Helsinki (1996). The research protocol was approved by the Lithuanian Bioethics Committee and an oral informed consent was obtained from all subjects. In total 5,405 women were approached; $79 \%$ of them agreed to participate in the study.

Exposures were assessed through questionnaires administered during pregnancy, requesting extensive information on personal characteristics and waterrelated habits. The first interview was completed during the first pregnancy trimester. The medium gestational age at the interview was 8 weeks. The interview queried women regarding demographics, residence and job characteristics, chronic diseases (cardiovascular, hypertension, diabetes, renal), reproductive history, including the date of last menstrual period, previous preterm delivery. We also asked the women to report their age (younger than 20 years, 20-29 years, 30 years, and over), educational level (primary, secondary, university), family status (married, not married), smoking (non-smoker, smoker at least one cigarette per day), alcohol consumption ( 0 drinks per week, at least one drink per week), blood pressure $(<140 / 90 \mathrm{~mm} / \mathrm{Hg}, \geq 140$ or $\geq 90 \mathrm{~mm} / \mathrm{Hg})$, body mass index $(<25 \mathrm{~kg} / \mathrm{m} 2,25-30 \mathrm{~kg} / \mathrm{m} 2,>30$ $\mathrm{kg} / \mathrm{m} 2$ ), and other potential risk factors for congenital anomalies. Adjustment for these variables was made for the studies of various subgroups. The women also were examined by ultrasound to determine the gestational age of the foetus.

A special questionnaire on water consumption and water use habits was used to interview 4,260 women who agreed to participate in the study; $76.4 \%$ of them were interviewed during the third pregnancy trimester before delivery at the hospital and $23.6 \%$ by telephone within the first month after delivery. Consumption was ascertained for three types of water: cold tape water or drinks made of cold tap water; boiled tap water (tea, coffee, and other); and bottled water, used at home, at work, etc. In addition, number of showers, baths, swimming pools weekly, and their average length was asked of all subjects. The interviews were conducted by trained nurses who did not know the THM exposure status and birth outcome.

Pregnancy outcomes were abstracted from the

medical records. We obtain registry-based data on any congenital anomalies from medical records. The birth defects studied were those reported in other studies and included cardiac anomalies, neural tube defects, cleft defects, chromosomal abnormalities, and others. The diagnoses of birth defects in this study were limited to those detectable before an infant is discharged from hospital after delivery. The reference group was defined as all term births (born at $>37$ weeks of gestation).

Women with multiple pregnancies (150), having inconsistent or invalid data for dating the pregnancy (5) or estimating THM exposure mostly students moved out of the city during pregnancy (839), parity more than 3 children (117) or with newborn birth weight above 4,500 g (75) were excluded. We restricted our analyses to infants born with a birth weight below 4,500 g, leaving the data for 3,074 women in the final analysis.

\subsection{THM exposure assessment}

The Kaunas city municipal drinking water is supplied by the four water treatment plants system. Groundwater sources are used for the whole water supply system. However, the four water treatment plants, which disinfected ground water with sodium hypochlorite (chlorine dose $0.26-0.91 \mathrm{mg} / \mathrm{L}$, residual chlorine $\quad 0-0.22 \mathrm{mg} / \mathrm{L})$, produce different concentrations of THMs in finished water. One treatment plant (Petraiunai) supplies finished water with higher levels of THMs ("high level THM site," $54.9 \%$ subjects), and the three other plants supply 
finished water with lower levels of all THMs ("low level THM site"). Water samples were collected four times per year over a 3-year study period (20072009) in the morning in three locations: close to the treatment plant, at $5 \mathrm{~km}$, and at $10 \mathrm{~km}$ or more from every treatment plant. A total of 85 water samples were collected from 12 monitoring sites in four water supply zones for THM analysis. Samples were analysed at the University of the Aegean, Greece, by using gas chromatography with electron captures detection (Nikolaou et al. 2005). Measurements included specific values for the four regulated THMs (total trihalomethane (TTHM), chloroform $(\mathrm{CH})$, bromodicholormethane (BDCM) and dibromochloromethane (DBCM). We calculated the mean quarterly THM constituent concentrations for water zones and, subsequently depending on the TTHM levels within each zone, assigned "low level" and "high level" sites. We used tap water THM concentration, derived as the average of quarterly sample values over the time that the pregnancy occurred from all sampling sites located in the each distribution system, and geocoded maternal address at birth to assign the individual women residential exposure index. Estimates of exposure index to total and specific THMs from drinking water were tabulated first as an average level at the tap over the pregnancy period; this measure was then categorised at the quartiles of the distribution for birth outcomes. We combined every subject's residential exposure index and water-use questionnaire data to assess individual exposure through ingestion of THMs. Women were asked to indicate the cup or glass size and number of cups or glasses of tap water consumed per day, including hot and cold beverages made from tap water. With this information, we calculated daily amounts of hot and cold tap water ingested. Integration of the information was carried out on residential THM levels $(\mu \mathrm{g} / \mathrm{L})$, ingested amounts (L/day) and modifications by heating using an estimated uptake factor of 0.00490 to derive an integrated index of blood concentration expressed in micrograms per day $(\mu \mathrm{g} / \mathrm{d})$ (Savitz et al. 2006; Whitaker et al. 2003).

The actual algorithms of internal dose from ingestion were:

- chloroform level $(\mu \mathrm{g} / \mathrm{L}) \times$ water consumption $(\mathrm{L} /$ day $) \times 0.00490196 \mu \mathrm{g} / \mu \mathrm{g} / \mathrm{L}$;

- brominated THM level $(\mu \mathrm{g} / \mathrm{L}) \times$ water consumption $(\mathrm{L} /$ day $) \times 0.00111848 \mu \mathrm{g} / \mu \mathrm{g} / \mathrm{L}$.

We assumed a null THM level for any bottled water consumption since chlorination and ozonation in local bottled water production were not used.

Finally, we addressed dermal absorption and inhalation by considering showering and bathing alone and combined with ingestion. We multiplied residential THM levels $(\mu \mathrm{g} / \mathrm{L})$ by frequency and average duration of bathing or showering per day (min/day) and calculated each mother's entire pregnancy average daily uptake of THM internal dose $(\mu \mathrm{g} / \mathrm{d})$. We derived indices of daily uptake by integrating THM concentrations, duration of bathing and showering reported in a questionnaire administered to study participants and estimated uptake factors of 0.001536 and 0.001321 of THMs in blood per minute per microgram from showering and bathing, respectively (Backer et al. 2000; Lynberg et al. 2001). The uptake factors of THMs individual constituents were assessed on the relative changes in blood levels after 10 minute exposure (after versus before ingestion $1 \mathrm{~L}$ of tap water, 10 minute showering, and 10 minute bathing). The actual algorithms of internal dose from showering and bathing were:

- $\min /$ day showering $\times \mu \mathrm{g} / \mathrm{L}$ chloroform in water $\times 0.001536261 \mu \mathrm{g} / \mathrm{min} / \mu \mathrm{g} / \mathrm{L}$,

- $\min /$ day showering $\times \mu \mathrm{g} / \mathrm{L}$ brominated THM in water $\times 0.001352065 \mu \mathrm{g} / \mathrm{min} / \mu \mathrm{g} / \mathrm{L}$,

- $\quad \min /$ day bathing $\times \mu \mathrm{g} / \mathrm{L}$ chloroform in water $\times$ $0.001320755 \mu \mathrm{g} / \mathrm{min} / \mu \mathrm{g} / \mathrm{L}$,

- min/day bathing $\times \mu \mathrm{g} / \mathrm{L}$ brominated THM in water $\times 0.00129571 \mu \mathrm{g} / \mathrm{min} / \mu \mathrm{g} / \mathrm{L}$

We then used average daily total uptakes in our analysis as continuous and categorised variables. We calculated quartiles of total THM internal dose. This gave first $(0.0025-0.0295 \mu \mathrm{g} / \mathrm{d})$, second $(0.0295-$ $0.1899 \mu \mathrm{g} / \mathrm{d})$, third $(0.1899-0.4492 \mu \mathrm{g} / \mathrm{d})$ and forth $(0.4492-2.404 \mu \mathrm{g} / \mathrm{d})$ quartiles for average TTHM uptake. To reduce exposure misclassification errors in the subsequent analysis, we used a subset of women who through the entire pregnancy did not change their address.

\subsection{Statistical analysis}

The data analysis compared the any congenital anomalies of exposed women to total THMs and specific THM constituents. We used logistic regression to estimate the adjusted odds ratios (ORs) and the 95-percent confidence intervals (CIs) for congenital anomalies and the various exposure indices. We categorized TTHM internal dose in quartiles and evaluated the possible relationship between increases in congenital anomalies risk for an increase in estimated TTHM internal dose. We ran multivariate logistic regression models for the TTHMs, CH, DBCM, and BDCM for the pregnancy. We also used multiple linear regressions for TTHM internal dose analysis as a continuous variable to evaluate the relationship, if any between congenital anomalies and every $0.1 \mu \mathrm{g} / \mathrm{d}$ increase in TTHM internal dose.

In the logistic regression models for congenital anomalies outcomes, using personal data of the pregnant women sample, we assessed a variety of potential confounders identified by univariate analysis. Further, we examined the association of THM exposure and congenital anomalies with a multivariable analysis controlling for the effect of major covariates that changed the adjusted ORs for THM by $10 \%$ or more. The adjusted congenital anomalies analyses included maternal education, age, body mass index, alcohol consumption, smoking, blood pressure, stress, foetus number, infant gender and premature birth. 
Table 1.

Mean THM levels $(\mu \mathrm{g} / L)$ by sampling site and water supply zone

\begin{tabular}{|l|l|l|l|l||}
\hline $\begin{array}{l}\text { Tap water sampling } \\
\text { sites }\end{array}$ & $\begin{array}{l}\text { TTHMs }^{\mathrm{c}} \\
\text { Mean }\left(\mathrm{SD}^{\mathrm{d}}\right)\end{array}$ & $\begin{array}{l}\text { CH } \\
\text { Mean (SD) }\end{array}$ & $\begin{array}{l}\text { DBCM } \\
\text { Mean (SD) }\end{array}$ & $\begin{array}{l}\text { BDCM Mean } \\
(\text { SD) }\end{array}$ \\
\hline All sites & $9.8(12.4)$ & $7.8(10.2)$ & $0.3(0.5)$ & $1.7(2.2)$ \\
Low THM level $^{\mathrm{a}}$ & $1.3(1.2)$ & $0.9(1.0)$ & $0.1(0.2)$ & $0.3(0.5)$ \\
High THM level $^{\mathrm{b}}$ & $21.9(10.9)$ & $17.7(9.0)$ & $0.5(0.6)$ & $3.6(2.1)$ \\
\hline
\end{tabular}

${ }^{\mathrm{a}}$ Viciunai, Eiguliai, Kleboniskis. ${ }^{\mathrm{b}}$ Petrasiunai.

${ }^{\mathrm{c}}$ TTHMs = total trihalomethanes: the sum of $\mathrm{CH}$ (chloroform), DBCM (dibromochloromethane), and BDCM (bromodichloromethane).

${ }^{\mathrm{d}} \mathrm{SD}=$ standard deviation.

\section{Results}

\subsection{Daily THM uptake}

The mean tap water THM level in the low level site from three water treatment plants was $1.3 \mu \mathrm{g} / \mathrm{L}$, and in the high level site (Petrasiunai) $21.9 \mu \mathrm{g} / \mathrm{L}$ (Table 1).

The highest proportion of THM made chloroform. Bromoform was below the limit of detection. There was little spatial and seasonal variability in the THMs levels within the high and low areas. The estimated individual total uptake of THMs ranged between 0.0025 and $2.404 \mu \mathrm{g} / \mathrm{d}$. The total $\mathrm{CH}$ uptake ranged between 0.0013 and $2.1328 \mu \mathrm{g} / \mathrm{d}$. Mothers supplied with water who had a higher $\mathrm{CH}$ concentration generally also had a higher total internal dose. Daily uptake of BDCM ranged between 0.0001 and 0.3359 $\mu \mathrm{g} / \mathrm{d}$ and DBCM ranged between 0 and $0.0683 \mu \mathrm{g} / \mathrm{d}$.

\subsection{Congenital anomalies risk factors}

The women recruited were predominantly Lithuanians in ethnic origin (97.5\%) and did not smoke (93.5\%) (Table 2).

The mean age was 28.4 years, and the women tended to be highly educated $(55.6 \%$ with a university degree). In general, mothers who were single, had preterm delivery, or reported a chronic stress delivered a higher proportion of congenital anomalies newborns.

Table 2. Distribution of Kaunas cohort study subjects for various characteristics among congenital anomalies cases and controls, crude odds ratios (OR) and 95\% confidence intervals (CI)

\begin{tabular}{|c|c|c|c|c|c|c|c|c|}
\hline \multirow[t]{2}{*}{ Risk factors } & \multicolumn{2}{|c|}{ All participants } & \multicolumn{2}{|c|}{ Cases } & \multicolumn{2}{|c|}{ Controls } & \multicolumn{2}{|c|}{$\begin{array}{c}\text { Crude } \\
\text { Odds ratios }\end{array}$} \\
\hline & No & $\%$ & No & $\%$ & No & $\%$ & OR & $95 \%$ CI \\
\hline $\begin{array}{l}\text { Maternal age } \\
<20 \text { years } \\
20-29 \text { years } \\
\geq 30 \text { years }\end{array}$ & $\begin{array}{c}94 \\
1895 \\
1085\end{array}$ & $\begin{array}{c}3.1 \\
61.6 \\
35.3\end{array}$ & $\begin{array}{c}3 \\
100 \\
68\end{array}$ & $\begin{array}{c}1.8 \\
58.5 \\
39.8\end{array}$ & $\begin{array}{c}91 \\
1795 \\
1017\end{array}$ & $\begin{array}{c}3.1 \\
61.8 \\
35.0\end{array}$ & $\begin{array}{c}1 \\
0.59 \\
1.20\end{array}$ & $\begin{array}{l}0.18-1.90 \\
0.87-1.65\end{array}$ \\
\hline $\begin{array}{l}\text { Family status* } \\
\text { Married } \\
\text { Not married } \\
\end{array}$ & $\begin{array}{c}2554 \\
520 \\
\end{array}$ & $\begin{array}{l}83.1 \\
16.9 \\
\end{array}$ & $\begin{array}{c}129 \\
42 \\
\end{array}$ & $\begin{array}{l}75.4 \\
24.6 \\
\end{array}$ & $\begin{array}{c}2425 \\
478 \\
\end{array}$ & $\begin{array}{l}83.5 \\
16.5 \\
\end{array}$ & $\begin{array}{c}1 \\
1.65 \\
\end{array}$ & $1.15-2.37$ \\
\hline $\begin{array}{l}\text { Maternal education } \\
\text { Primary school } \\
\text { Secondary school } \\
\text { University degree } \\
\end{array}$ & $\begin{array}{c}147 \\
1217 \\
1710 \\
\end{array}$ & $\begin{array}{c}4.8 \\
39.6 \\
55.6\end{array}$ & $\begin{array}{c}9 \\
57 \\
105 \\
\end{array}$ & $\begin{array}{c}5.3 \\
33.3 \\
61.4 \\
\end{array}$ & $\begin{array}{c}138 \\
1160 \\
1605\end{array}$ & $\begin{array}{c}4.8 \\
40.0 \\
55.3 \\
\end{array}$ & $\begin{array}{c}1 \\
0.75 \\
1.00\end{array}$ & $\begin{array}{l}0.37-1.56 \\
0.50-2.03 \\
\end{array}$ \\
\hline $\begin{array}{l}\text { Maternal active smoking } \\
\text { Non-smoker } \\
\text { Smoker }\end{array}$ & $\begin{array}{c}2874 \\
200 \\
\end{array}$ & $\begin{array}{c}93.5 \\
6.5 \\
\end{array}$ & $\begin{array}{c}162 \\
9 \\
\end{array}$ & $\begin{array}{c}94.7 \\
5.3 \\
\end{array}$ & $\begin{array}{c}2712 \\
191 \\
\end{array}$ & $\begin{array}{c}93.4 \\
6.6 \\
\end{array}$ & $\begin{array}{c}1 \\
0.79 \\
\end{array}$ & $0.40-1.56$ \\
\hline $\begin{array}{l}\text { Passive smoking } \\
\text { Non-smoker } \\
\text { Smoker } \\
\end{array}$ & $\begin{array}{l}1664 \\
1378 \\
\end{array}$ & $\begin{array}{l}54.7 \\
45.3 \\
\end{array}$ & $\begin{array}{l}82 \\
86 \\
\end{array}$ & $\begin{array}{l}48.8 \\
51.2 \\
\end{array}$ & $\begin{array}{l}1582 \\
1292 \\
\end{array}$ & $\begin{array}{l}55.0 \\
45.0 \\
\end{array}$ & $\begin{array}{c}1 \\
1.28 \\
\end{array}$ & $0.94-1.75$ \\
\hline $\begin{array}{l}\text { Alcohol consumption } \\
\text { No } \\
\text { Yes }\end{array}$ & $\begin{array}{c}2889 \\
185 \\
\end{array}$ & $\begin{array}{c}94.0 \\
6.0 \\
\end{array}$ & $\begin{array}{c}165 \\
6 \\
\end{array}$ & $\begin{array}{c}96.5 \\
3.5 \\
\end{array}$ & $\begin{array}{c}2729 \\
179 \\
\end{array}$ & $\begin{array}{c}93.8 \\
6.2 \\
\end{array}$ & $\begin{array}{c}1 \\
0.55 \\
\end{array}$ & $0.24-1.27$ \\
\hline $\begin{array}{l}\text { Blood pressure* } \\
\quad<140 / 80 \mathrm{~mm} / \mathrm{Hg} \\
\geq 140 \text { or } \geq 90 \mathrm{~mm} / \mathrm{Hg}\end{array}$ & $\begin{array}{c}2643 \\
431 \\
\end{array}$ & $\begin{array}{l}86.0 \\
14.0 \\
\end{array}$ & $\begin{array}{c}157 \\
14 \\
\end{array}$ & $\begin{array}{c}91.8 \\
8.2 \\
\end{array}$ & $\begin{array}{c}2486 \\
417 \\
\end{array}$ & $\begin{array}{l}85.6 \\
14.4 \\
\end{array}$ & $\begin{array}{c}1 \\
0.53 \\
\end{array}$ & $0.31-0.93$ \\
\hline $\begin{array}{l}\text { Ethnic group } \\
\text { Lithuanian } \\
\text { Other } \\
\end{array}$ & $\begin{array}{c}2997 \\
77 \\
\end{array}$ & $\begin{array}{c}97.5 \\
2.5 \\
\end{array}$ & $\begin{array}{c}168 \\
3 \\
\end{array}$ & $\begin{array}{c}98.2 \\
1.8 \\
\end{array}$ & $\begin{array}{c}2829 \\
74 \\
\end{array}$ & $\begin{array}{c}97.5 \\
2.5 \\
\end{array}$ & $\begin{array}{c}1 \\
0.68 \\
\end{array}$ & $0.21-2.19$ \\
\hline $\begin{array}{l}\text { Parity } \\
\text { No child } \\
\geq 1 \text { child } \\
\end{array}$ & $\begin{array}{l}1515 \\
1559 \\
\end{array}$ & $\begin{array}{l}49.3 \\
50.7 \\
\end{array}$ & $\begin{array}{l}86 \\
85 \\
\end{array}$ & $\begin{array}{l}50.3 \\
49.7 \\
\end{array}$ & $\begin{array}{l}1429 \\
1474 \\
\end{array}$ & $\begin{array}{l}49.2 \\
50.8 \\
\end{array}$ & $\begin{array}{c}1 \\
0.96 \\
\end{array}$ & $0.70-1.30$ \\
\hline Infant gender & & & & & & & & \\
\hline
\end{tabular}




\begin{tabular}{|c|c|c|c|c|c|c|c|c|}
\hline \multirow[t]{2}{*}{ Risk factors } & \multicolumn{2}{|c|}{ All participants } & \multicolumn{2}{|c|}{ Cases } & \multicolumn{2}{|c|}{ Controls } & \multicolumn{2}{|c|}{$\begin{array}{c}\text { Crude } \\
\text { Odds ratios }\end{array}$} \\
\hline & No & $\%$ & No & $\%$ & No & $\%$ & OR & $95 \% \mathrm{CI}$ \\
\hline Male & 1558 & 50.7 & 91 & 53.2 & 1467 & 50.5 & 1 & \\
\hline Female & 1516 & 49.3 & 80 & 46.8 & 1436 & 49.5 & 0.90 & $0.66-1.22$ \\
\hline \multicolumn{9}{|l|}{ Current residence } \\
\hline 2-4 years & 1301 & 42.3 & 72 & 41.7 & 1229 & 42.3 & 1 & \\
\hline 5-9 years & 763 & 24.8 & 43 & 25.1 & 720 & 24.8 & 1.02 & $0.69-1.50$ \\
\hline$\geq 10$ years & 1010 & 32.9 & 56 & 32.7 & 954 & 32.9 & 1.00 & $0.70-1.44$ \\
\hline \multicolumn{9}{|l|}{ Work exposure } \\
\hline No & 1089 & 35.4 & 55 & 32.2 & 1034 & 35.6 & 1 & \\
\hline Yes & 1985 & 64.6 & 116 & 67.8 & 1869 & 64.4 & 1.17 & $0.84-1.62$ \\
\hline \multicolumn{9}{|l|}{ Chronic disease } \\
\hline No & 2333 & 75.9 & 125 & 73.1 & 2208 & 76.1 & 1 & \\
\hline Yes & 741 & 24.2 & 46 & 26.9 & 695 & 23.9 & 1.17 & $0.83-1.66$ \\
\hline \multicolumn{9}{|l|}{ Previous preterm delivery } \\
\hline No & 3023 & 98.3 & 167 & 97.7 & 2856 & 98.4 & 1 & \\
\hline Yes & 51 & 1.7 & 4 & 2.3 & 47 & 1.6 & 0.48 & $0.52-4.09$ \\
\hline \multicolumn{9}{|l|}{ Socio economic status } \\
\hline Low income & 906 & 30.6 & 52 & 31.7 & 854 & 30.6 & 1 & \\
\hline Medium income & 1594 & 53.9 & 78 & 47.6 & 1516 & 54.2 & 0.85 & $0.59-1.21$ \\
\hline High income & 459 & 15.5 & 34 & 20.7 & 425 & 15.2 & 1.31 & $0.84-2.06$ \\
\hline \multicolumn{9}{|l|}{ Body mass index $\left(\mathrm{kg} / \mathrm{m}^{2}\right)^{*}$} \\
\hline$<25$ Normal & 1812 & 58.9 & 127 & 74.3 & 1685 & 58.0 & 1 & \\
\hline 25-30 Overweight & 866 & 28.2 & 27 & 15.8 & 839 & 28.9 & 0.43 & $0.28-0.65$ \\
\hline 30 Obesity & 396 & 12.9 & 17 & 9.9 & 379 & 13.1 & 0.60 & $0.35-1.00$ \\
\hline \multicolumn{9}{|l|}{ Water filter } \\
\hline Yes & 931 & 30.3 & 53 & 31.0 & 878 & 30.2 & 1 & \\
\hline No & 2143 & 69.7 & 118 & 69.0 & 2025 & 69.8 & 0.97 & $0.69-1.35$ \\
\hline \multicolumn{9}{|l|}{ Water supply area } \\
\hline Petrasiunai & 1681 & 54.7 & 103 & 60.2 & 1578 & 54.4 & 1 & \\
\hline Other & 1693 & 45.3 & 68 & 39.8 & 1325 & 45.6 & 1.27 & $0.93-1.74$ \\
\hline \multicolumn{9}{|l|}{ Gestation age* } \\
\hline$\geq 37$ weeks & 2851 & 92.7 & 147 & 86.0 & 2704 & 93.1 & 1 & \\
\hline$<37$ weeks & 223 & 7.3 & 24 & 14.0 & 199 & 6.9 & 2.22 & $1.41-3.50$ \\
\hline \multicolumn{9}{|l|}{ Maternal stress* } \\
\hline No & 2588 & 84.2 & 132 & 77.2 & 2456 & 84.6 & 1 & \\
\hline Yes & 486 & 15.8 & 39 & 22.8 & 447 & 15.4 & 1.62 & $1.12-2.35$ \\
\hline
\end{tabular}

$*: p<0.05$

\subsection{Association between THM internal dose during pregnancy and congenital anomalies risk}

Using total gestational daily uptakes quartiles of TTHM and individual THMs continuous variables, we examined the association between internal dose for the entire pregnancy and any congenital anomalies risk (Table 3). Effect estimates based on TTHMs and $\mathrm{CH}$ quartiles tended to increase by an increase in the exposure quartiles compared with the first quartile, but there were no statistically significant effect for entire pregnancy. TTHM and $\mathrm{CH}$ analysed as continuous variables (increase in $0.1 \mu \mathrm{g} / \mathrm{d}$ ) also showed slightly elevated, but statistically nonsignificant increase in risk of any congenital anomalies. However, we found dose-response relationships for the BDCM internal dose and risk for any congenital anomalies for crude and adjusted ORs. The adjusted ORs for fourth quartile vs. first quartile was $1.5,95 \%$ CI $1.00-2.55$. The ORs per every 0.01 $\mu \mathrm{g} / \mathrm{d}$ increase in BDCM internal dose was $1.03,95 \%$ CI 1.00-1.07. Effect estimates from continuous model associated with DBCM exposure levels during entire pregnancy also revealed statistically significant association with the any congenital anomalies risk (OR 1.23, 95\% CI 1.02-1.48).

\section{Discussion}

Assessment of potential effects of exposure to drinking water disinfection by-products on the risk of congenital anomalies is problematic because of rarity and diversity of the congenital malformations.

In this study the outcome assessment was based on birth registration, as in the vast majority of the previous studies of disinfection by-products and birth defects. However, THM exposure assessment has included personal exposure estimation through multiple routes such as bathing, showering and water consumption. Also we have personal information on alcohol consumption, cigarette smoking, other behaviour factors, residential history, mobility during pregnancy and other variables whose influence we controlled during assessment impact of individual THM constituents exposure on congenital anomalies.

We conducted a prospective epidemiological study to examine the effects of internal dose of THM during the entire pregnancy on the risk of any 
congenital anomalies. We found little indication of a dose-response relationship for total THM and $\mathrm{CH}$ and congenital anomalies. Total THMs and $\mathrm{CH}$ analysed as continuous variables (increase in $0.1 \mu \mathrm{g} / \mathrm{d}$ ) showed slightly elevated, but statistically non-significant increase in risk of any congenital anomalies. For the BDCM association with the any congenital anomalies the dose-response relationship was evident for entire pregnancy. The adjusted ORs for fourth quartile vs. first quartile was $1.5,95 \%$ CI $1.00-2.55$ and the probability of delivering any congenital anomalies infant was elevated by $3 \%$ per every $0.01 \mu \mathrm{g} / \mathrm{d}$ increase in the pollutant internal dose (OR 1.03, 95\% CI 1.00-1.07). Effect estimates from continuous model associated with DBCM exposure levels during entire pregnancy also revealed statistically significant association with the any congenital anomalies risk (OR 1.23, 95\% CI 1.02-1.48). The lack of statistically significant effects for other THMs constituents may be due to low exposure because of low THM levels and lack of power in our study sample.

Table 3. Congenital anomalies crude and adjusted odds (OR) ratios and 95\% confidence intervals (CI) for entire pregnancy exposure to internal dose THM quartiles

\begin{tabular}{|c|c|c|c|c|}
\hline $\begin{array}{l}\text { THM dose quartiles } \\
\text { limits }(\mu \mathrm{g} / \mathrm{d})\end{array}$ & $\begin{array}{l}\text { Cases } \\
\mathrm{N}(\%)\end{array}$ & $\begin{array}{l}\text { Controls } \\
\mathrm{N}(\%) \\
\end{array}$ & $\begin{array}{c}\text { Crude } \\
\text { OR }(95 \% \mathrm{CI}) \\
\end{array}$ & $\begin{array}{c}\text { Adjusted* } \\
\text { OR }(95 \% \mathrm{CI})\end{array}$ \\
\hline \multicolumn{5}{|l|}{ THM } \\
\hline $0.0025-0.0295$ & $35(4.6)$ & $726(95.4)$ & 1 & 1 \\
\hline $0.0295-0.1899$ & $39(4.9)$ & $752(95.1)$ & $1.08(0.67-1.72)$ & $1.05(0.65-1.69)$ \\
\hline $0.1899-0.4492$ & $45(5.8)$ & $731(94.2)$ & $1.28(0.81-2.01)$ & $1.28(0.81-2.02)$ \\
\hline $0.4492-2.4040$ & $52(7.0)$ & $694(93.0)$ & $1.55(1.00-12.42)$ & $1.45(0.92-2.27)$ \\
\hline \multicolumn{3}{|l|}{ Continuous $(0.1 \mu \mathrm{g} / \mathrm{d})$} & & $1.03(0.98-1.08)$ \\
\hline \multicolumn{5}{|l|}{ Chloroform } \\
\hline $0.0013-0.1807$ & $38(5.0)$ & $724(95.0)$ & 1 & 1 \\
\hline $0.1807-0.1457$ & $36(4.6)$ & $753(5.4)$ & $0.91(0.57-1.45)$ & $0.89(0.56-1.43)$ \\
\hline $0.1457-0.3707$ & $47(6.0)$ & $730(94.0)$ & $1.23(0.79-1.90)$ & $1.22(0.78-1.91)$ \\
\hline $0.3707-2.1328$ & $50(6.7)$ & $696(93.3)$ & $1.37(0.89-2.11)$ & $1.27(0.82-1.99)$ \\
\hline \multicolumn{3}{|l|}{ Continuous $(0.1 \mu \mathrm{g} / \mathrm{d})$} & & $1.02(0.97-1.08)$ \\
\hline \multicolumn{5}{|l|}{ BDCM } \\
\hline $0.0001-0.01$ & $31(4.0)$ & $742(96.0)$ & 1 & 1 \\
\hline $0.01-0.0291$ & $45(5.8)$ & $732(94.2)$ & $1.47(0.92-2.35)$ & $1.44(0.89-2.32)$ \\
\hline $0.0291-0.0609$ & $43(5.7)$ & $710(94.3)$ & $1.45(0.90-2.33)$ & $1.49(0.92-2.41)$ \\
\hline $0.0609-0.3359$ & $52(6.7)$ & $719(93.3)$ & $1.73(1.10-2.73)$ & $1.59(1.00-2.55)$ \\
\hline \multicolumn{3}{|l|}{ Continuous $(0.01 \mu \mathrm{g} / \mathrm{d})$} & & $1.03(1.00-1.07)$ \\
\hline \multicolumn{5}{|l|}{ DBCM } \\
\hline $0.0000-0.00101$ & $37(5.2)$ & $674(94.8)$ & 1 & 1 \\
\hline $0.00101-0.00267$ & $37(4.8)$ & $738(95.2)$ & $0.91(0.57-1.46)$ & $0.94(0.59-1.52)$ \\
\hline $0.00267-0.00809$ & $41(5.2)$ & $750(94.8)$ & $1.00(0.63-1.57)$ & $1.00(0.63-1.59)$ \\
\hline $0.00809-0.0683$ & $56(7.0)$ & $741(93.0)$ & $1.38(0.90-2.11)$ & $1.32(0.85-2.05)$ \\
\hline \multicolumn{3}{|l|}{ Continuous $(0.01 \mu \mathrm{g} / \mathrm{d})$} & & $1.23(1.02-1.48)$ \\
\hline
\end{tabular}

*Adjusted for maternal education, age, body mass index, alcohol consumption, passive smoking, blood pressure, stress, foetus number, infant gender and premature birth.

TTHM, total trihalomethane; DBCM-dibromochloromethane, BDCM-bromodichloromethane

\section{Discussion}

Assessment of potential effects of exposure to drinking water disinfection by-products on the risk of congenital anomalies is problematic because of rarity and diversity of the congenital malformations.

In this study the outcome assessment was based on birth registration, as in the vast majority of the previous studies of disinfection by-products and birth defects. However, THM exposure assessment has included personal exposure estimation through multiple routes such as bathing, showering and water consumption. Also we have personal information on alcohol consumption, cigarette smoking, other behaviour factors, residential history, mobility during pregnancy and other variables whose influence we controlled during assessment impact of individual THM constituents exposure on congenital anomalies.

We conducted a prospective epidemiological study to examine the effects of internal dose of THM during the entire pregnancy on the risk of any congenital anomalies. We found little indication of a dose-response relationship for total THM and $\mathrm{CH}$ and congenital anomalies. Total THMs and $\mathrm{CH}$ analysed as continuous variables (increase in $0.1 \mu \mathrm{g} / \mathrm{d}$ ) showed slightly elevated, but statistically non-significant increase in risk of any congenital anomalies. For the BDCM association with the any congenital anomalies the dose-response relationship was evident for entire pregnancy. The adjusted ORs for fourth quartile vs. first quartile was $1.5,95 \%$ CI $1.00-2.55$ and the probability of delivering any congenital anomalies infant was elevated by $3 \%$ per every $0.01 \mu \mathrm{g} / \mathrm{d}$ increase in the pollutant internal dose (OR 1.03, 95\% CI 1.00-1.07). Effect estimates from continuous model associated with DBCM exposure levels during entire pregnancy also revealed statistically significant association with the any congenital anomalies risk (OR 1.23, 95\% CI 1.02-1.48). The lack of statistically significant effects for other THMs constituents may 
be due to low exposure because of low THM levels and lack of power in our study sample.

Reconciling our results with previous findings is not straightforward because there are substantial differences in the THMs levels, individual THMs constituents in drinking water, measurement of personal exposures, its classification, variation of exposure during pregnancy and controlling for confounding variables. Also the vast majority of epidemiological studies for the exposure assessment use THM concentration in drinking water as an exposure index without estimation of personal water consumption habits and estimation of THM uptake. These shortcomings may lead to the misclassification of exposure.

Only few previous studies have focused on the study associations between THM exposure and the risk of all the most common congenital anomalies. The authors of a nationwide cross-sectional study of Norwegian births (Hwang et al. 2002) to assess the effect of water chlorination by-products on congenital anomalies compared risks of birth defects according to four exposure categories. The exposure categories were estimated on the basis of chlorination (yes/no) and the level of water colour, representing the amount of natural organic matter. In logistic regression analysis, the risks of any congenital anomalies were significantly associated with increased exposure (OR $1.13,95 \%$ CI $1.01-1.25)$. In a registry-based study to determine the relationship between THM concentrations and the risk of congenital anomalies in England and Wales (Nieuwenhuijsen et al. 2008) THM data were obtained from water companies. In this large national study the authors found little evidence for a relationship between THM concentrations in drinking water and risk of any congenital anomalies. A population-based crosssectional Taiwanese births study (Hwang et al. 2008) compared the risk of congenital anomalies in four THM exposure categories, based on the levels of total THM. The study results showed no consistent association between exposure and the risk of any congenital anomalies in general.

A major challenge of these studies is the imprecision of exposure assessment from using aggregate municipal measures for classifying THM exposures. These studies also have no information on the amounts of beverage and tap water consumption by pregnant women and exposure to volatile disinfection by-products through inhalation and dermal absorption, which introduce non-differential misclassification and decrease the accuracy of exposure assessment. The studies have no information on residential mobility during pregnancy that may have produced exposure classification errors.

An epidemiological Australian communities study reported a statistically significant increased risk of any congenital anomalies (OR 1.22, 95\% CI 1.011.48) among women users of high levels of TTHMs in drinking water with the highest proportion (on average, $92 \%$ ) of brominated THMs (Chisholm et al. 2008). These results are consistent with our data that the highest effect of total THMs on congenital anomalies comes from brominated THMs.

The strengths of our study include the THM exposure assessment during pregnancy, a populationbased assessment of cases and controls, control for residential mobility effects. This study also offers advancement in individual internal dose calculation based on residential THM levels, detailed water use behaviours and exposure during pregnancy. Every subject's exposure indices were estimated as daily internal dose of the THM constituents ( $\mu \mathrm{g} / \mathrm{d})$, and birth outcome effects were assessed by using indices categorical variable and also as a continuous variable. An additional strength of our study is that pregnant women were prospectively followed, and did not move during pregnancy. That allowed collection of self reported data on potential confounding covariates and decrease in exposure misclassification errors.

We acknowledge several limitations in this study. Because of the lack of information regarding the validity of the internal dose assessment models that we used in our study, there is a possibility that the effect estimates that we have observed may be biased owing to non-differential misclassification of internal dose. Also we have not studied epigenetic and genetic interaction as part of the mechanism participating in metabolism of environmental contaminants.

Further epidemiological studies to determine whether an association exists between exposure to THMs and developmental effects must consider the individual THM, its concentrations, since the mechanisms of toxic action differ in individual THMs compounds (Bull et al. 2009; Richardson et al. 2007; Health Canada 2006). The influence of the water distribution system structure and the spatial variability of DBPs on the population exposure to water disinfection by-products also should be accounted for the exposure assessment (Legay et al. 2010). A recent workshop highlighted the current challenges and new opportunities for studying the role of genetic factors in the etiology of human birth defects (Olshan et al. 2011). In future studies personal estimation of the uptake substances and research into geneenvironmental interaction could be a challenge.

Given the controversy surrounding THM levels in drinking water and adverse pregnancy outcomes, especially regarding associated congenital anomalies, a precautionary approach to brominated THM exposure during pregnancy is somewhat justified.

\section{Conclusions}

The present study suggests that prenatal exposure to drinking water THM may increase the risk of any congenital anomalies. Our findings demonstrate the importance of focusing on exposure of individual THM constituents at the personal level of the studies' impact on pregnancy outcomes, rather than using total THM concentrations in drinking water. A precautionary approach to brominated THM exposure during pregnancy should be used to decrease risk of congenital anomalies. 


\section{Acknowledgment}

This work was supported in part by grant FP6036224 from the European Commission. We acknowledge the contribution of the Clinics of Kaunas University of Medicine involved in registration of pregnancy outcomes. We also thank Maria Kostopoulou-Karadanelli and Stuart W Krasner for THM analyses and consultations.

\section{References}

BACKER, L. C., ASHLEY, D. L., BONIN, M. A., CARDINALI, F. L., KIESZAK, S. M., WOOTEN, J. V. Household exposures to drinking water disinfection byproducts: whole blood trihalomethane levels. Journal of Exposure Analysis and Environmental Epidemiology, 2000, 10(4). pp. 321-326. http://dx.doi.org/10.1038/sj.jea.7500098

BOVE, F., SHIM, Y., ZEITZ, P. Drinking water contaminants and adverse pregnancy outcomes: a review. Environmental Health Perspective, 2002, Vol. 110 (No. S11). pp. 61-74.

BULL, R., RICE, G., TEUSCHLER, L., FEDER, P. Chemical Measures of Similarity Among Disinfection ByProduct Mixtures. J Toxicol Env Heal A, 2009, 72. pp. 482493. http://dx.doi.org/10.1080/15287390802608973

BUTTERWORTH, B. E. Science-based risk assessments for drinking water disinfection by-products. Environ Res, 2005, Vol. 98, 2. pp. 276-278.

CEDERGREN, M. I., SELBING, A. J., LÖFMAN, O., KÄLLEN, B. A. Chlorination byproducts and nitrate in drinking water and risk for congenital cardiac defects. Environ Res, 2002, 89. pp. 124-130. http://dx.doi.org/10.1006/enrs.2001.4362

CHISHOLM, K., COOK, A., BOWER, C., WEINSTEIN, P. Risk of birth defects in Australian communities with high brominated disinfection by-product levels. Environ. Health Perspect. 2008, 116(9). pp 12671273. http://dx.doi.org/10.1289/ehp.10980

GOEBELL, P. J., VILLANUEVA, C. M. RETTENMEIER, A. W., RUBBEN, H., KOGEVINAS, M. Environmental exposure, chlorinated drinking water, and bladder cancer. World J Urol, 2004, Vol. 21, 6. pp. 424 432 .

GRAZULEVICIENE, R., NIEUWENHUIJSEN, M J., VENCLOVIENE, J., KOSTOPOULOUKARADANELLI, M., KRASNER, S. W., DANILEVICIUTE, A., BALCIUS, G., KAPUSTINSKIENE, V. Individual exposures to drinking water trihalomethanes, low birth weight and small for gestational age risk: a prospective Kaunas cohort study. Environmental Health, 2011, 10. pp. 1-32. http://dx.doi.org/10.1186/1476-069X-10-32

HEALTH CANADA. Guidelines for Canadian Drinking Water Quality: Guideline Technical Document Trihalomethanes. Ottawa, ON.: Health Canada, 2006, 70.
HWANG, B. F., JAAKKOLA, J. J. K. A systematic review and meta-analysis.Arch Environ Health, 2003, 58. pp. 83-91. http://dx.doi.org/10.3200/AEOH.58.2.83-91

HWANG B. F, JAAKKOLA, J. J. K., GUO H. R. Water disinfection by-products and the risk of specific birth defects: a population-based cross-sectional study in Taiwan. Environ. Health, 2008, 2. pp. 7-23. http://dx.doi.org/10.1093/aje/kwf038

HWANG, B. F., MAGNUS, P., JAAKKOLA, J. J. K. Risk of specific birth defects in the relation to chlorination and among of the natural organic matter in the water supply. Am J Epidemiol, 2002, 156. pp. 374-382.

LEGAY, C. H., RODRIQUEZ, M. J., SERODES, LEVALLOIS, P. The assessment of population exposure to chlorination by-products: a study on the influence of the water distribution system. Environmental Health, 2010, 9, 59doi:10. pp. 1186/1476-069X-9-59.

LYNBERG, M., NUCKOLS, J. R., LANGLOIS, P., ASHLEY, D., SINGER, P., MENDOLA, P., WILKES, C., KRAPFL, H., MILES, E., SPEIGHT, V., LIN, B., SMALL, L., MILES, A., BONIN, M., ZEITZ, P., TADKOD, A., HENRY, J., FORRESTER, M. B. Assessing exposure to disinfection by-products in women of reproductive age living in Corpus Christi, Texas, and Cobb County, Georgia: descriptive results and methods. Environmental Health Perspectives, 2001, 109(6). pp. 597-604. http://dx.doi.org/10.1289/ehp.01109597

NIEUWENHUIJSEN, M. J., GRELLIER, J., SMITH, R., ISZATT, N., BENNETT, J., BEST, N., TOLEDANO, M. The epidemiology and possible mechanisms of disinfection by-products in drinking water. Philosophical Transaction. Series A, Mathemathical, Physical and Enginieric Science, 2009, Vol. 367, No. 1904. pp. 40434076.

NIEUWENHUIJSEN, M. J., TOLEDANO, M. B., BENNETT, J., BEST, N., HAMBLY, P., DE HOOGH, C., WELLESLEY, D., BOYD, P. A., ABRAMSKY, L., DATTANI, N., FAWELL, J., BRIGGS, D., JARUP, L., HELLIOTT, P. Chlorination disinfection by-products and risk of congenital anomalies in England and Wales. Environ Health Perspect, 2008, 16. pp. 216-222.

NIEUWENHUIJSEN, M. J., TOLEDANO, M. B., EATON, N. E., FAWELL, J., ELLIOTT, P. Chlorination disinfection by-products in water and their association with adverse reproductive outcomes: a review. Occup Environ Med, 2000, Vol. 57. pp. 73-85. http://dx.doi.org/10.1136/oem.57.2.73

NIKOLAOU, A., GOLFINOPOULOS, S., RIZZO, L., LOFRANO, G., LEKKAS, T., BELGIORNO, V. Optimisation of analytical methods for the determination of DBPs: Application to drinking waters from Greece and Italy. Desalination, 2005, Vol. 176, No. 1-3, pp. 25-36. http://dx.doi.org/10.1016/j.desal.2004.10.028

OLSHAN, A. F., HOBBS, C. A., SHAW, G. M. Discovery of genetic susceptibility factors for human birth defects: an opportunity for a National Agenda. Am J Med Genet A, 2011, 155A(8). pp. 1794-1797. http://dx.doi.org/10.1002/ajmg.a.34103

RICHARDSON, S. D., PLEWA, M. J., WAGNER, E. D., SCHOENY, R., DEMARINI, D. M. Occurence, 
genotoxicity, and carcinogenicity of regulated and emerging disinfection by-products in drinking water: A review and roadmap for research. Mutation Research, 2007, 636. pp. 178-242. http://dx.doi.org/10.1016/j.mrrev.2007.09.001

SAVITZ, D. A., SINGER, P. C., HERRING, A. H., HARTMANN, K. E., HOWARD, S., WEINBERG, H. S., MAKARUSHKA, C. Exposure to drinking water disinfection by-products and pregnancy loss. American Journal of Epidemiology, 2006, 164(11). pp. 1043-1051. http://dx.doi.org/10.1093/aje/kwj300

SHAW, G. M., RANATUNGA, D., QUACH, T., NERI, E., CORREA, A., NEUTRA, R. R. Trihalomethane exposures from municipal water supplies and selected congenital malformations. Epidemiology, 2003, 14. pp. 191-199.

http://dx.doi.org/10.1097/01.EDE.0000050697.18634.A6

TARDIFF, R. G., CARSON, M. L., GINEVAN, M. E. Updated weight of evidence for an association between adverse reproductive and developmental effects and exposure to disinfection by-products. Regulatory Toxicology and Pharmacology, 2006, Vol. 45, No. 2. pp. 185-205. http://dx.doi.org/10.1016/j.yrtph.2006.03.001

WHITAKER, H. J., NIEUWENHUIJSEN, M. J., BEST, N. G. The relationship between water concentrations and individual uptake of chloroform: a simulation study.
Environmental Health Perspective, 2003, 111(5). pp. 688694. http://dx.doi.org/10.1289/ehp.5963

MSc. Violeta Kapustinskienė, $\mathrm{PhD}$ student at the Department of Environmental Sciences Faculty of Natural Sciences of Vytautas Magnus University.

Main research areas: environmental epidemiology, health risks assessment.

Address: $\quad$ Vileikos str.8,

44404 Kaunas, Lithuania

Tel.: $\quad+37037327904$

Fax: $\quad+37037327904$

E-mail: $\quad$ Violeta.Kapustinskiene@gmf.vdu.lt

Prof. habil.dr. Regina Gražulevičienė, Professor at the Department of Environmental Sciences Faculty of Natural Sciences of Vytautas Magnus University.

Main research areas: environmental epidemiology, environmental exposures estimation, health risks assessment.

Address: $\quad$ Vileikos str.8,

44404 Kaunas, Lithuania

Tel.: $\quad+37037734642$

Fax: $\quad+37037327904$

E-mail: $\quad$ Regina.Grazuleviciene@gmf.vdu.lt

\title{
Vandens dezinfekcijos pašaliniai produktai ir igimtų anomalijų rizika Kaune
}

\author{
Violeta Kapustinskienė, Regina Gražulevičienė
}

Vytauto Didžiojo universitetas

(gauta 2012 m. vasario mèn., priimta spaudai 2012m. rugsèjo mèn.)

Remiantis epidemiologiniu tyrimu duomenimis, motinos ekspozicija geriamojo vandens chloravimo pašaliniais produktais trihalometanais (THM) gali būti susijusi su igimtomis anomalijomis. Šio tyrimo tikslas buvo nustatyti motinos ekspozicijos ị atskirų THM vidinès dozès nèštumo metu poveikị igimtų anomalijų rizikai. Buvo atliktas populiacinis perspektyvusis tyrimas, i kuri 2007-2009 m. itrauktos 3074 nëščiosios kaunietès, kurioms ekspozicijai nustatyti buvo apskaičiuota THM vidinė dozė. Naudojant daugiaveiksnę logistinę regresiją, keturiuose vidinės dozès ekspozicijos kvartiliuose palyginta ịgimtų anomalijų rizika. Be to, taikant tiesinès regresijos modelius kontroliuota moterų fiziologinių, elgsenos, socialinių ir demografinių veiksnių itaka.

Visų dèl THM ir chloroformo ịgimtu anomalijų rizika didejjant ekspozicijos kvartiliui turèjo tendenciją didèti. Nustatytas ryšys tarp bromodichlorometano (BDCM) vidinès dozės ir igimty anomaliju rizikos. Standartizuotas galimybiu santykis (GS) tarp pirmo ir ketvirto kvartilio buvo $1,5,95 \%$ PI 1,00-2,55, o padidejjus $0,01 \mu \mathrm{g} / \mathrm{d}$ BDCM vidinei dozei reikšmingai padidèjo igimtu anomaliju rizika (GS 1,03, 95\% PI 1,00-1,07). Dibromochlorometano (DBCM) ekspozicija taip pat reikšmingai didino igimtų anomalijų riziką (GS 1,23, 95\% PI 1,02-1,48).

Gauti tyrimai rodo, kad dèl visu THM igimtu anomaliju yra tendencija, kad rizika gali didèti, tačiau esant BDCM ir DBCM ekspozicijai statistiškai reikšmingai didejja ịgimtų anomalijų rizika. 